\title{
Steady State Electro-Thermal Modeling For DC-DC Converters ${ }^{1}$
}

\author{
Rosa Ciprian, Brad Lehman \\ Northeastern University \\ Department of Electrical \& Computer Engineering \\ Boston, MA 02115, USA \\ Email : ciprian.r@ece.neu.edu
}

\begin{abstract}
This paper presents a method to combine CFD simulation with electrical simulation packages. This approach leads to highly accurate electro-thermal system modeling for DC-DC converters. Based on an initial power loss of main components and a thermal model of the converter, a user friendly CFD thermal simulation is used to obtain an initial point for case steady-state temperatures. These set of temperatures are then fed into temperature-dependent electrical models and simulations. The procedure can be repeated while error levels remain within an acceptable range. This approach can lead to a relatively accurate electro-thermal model at steady state.
\end{abstract}

\section{INTRODUCTION}

Most consumer electronics have a traditional maximum operating temperature of $70^{\circ} \mathrm{C}$, however, harsh environment applications, such as those found in defense, automotive and oil exploration systems have higher maximum operating temperatures and require more sophisticated and capable electronics. In such applications, power converters usually face conditions of uncontrolled ambient temperature and space limitations. Moreover, converters usually have to be contained in a sealed enclosure in order to assure safety and noise immunity. Thermal management of these systems is required to achieve a successful and reliable design that meets high temperature environmental and reliability requirements [1].

Various approaches have been proposed for reducing thermal stress in electronic components. Uniformly, each approach attempts to manipulate the thermal conduction and convection rate of the environment surrounding the power components in order to keep them within their thermal limits [2]. Some methods are based on improving the heat flow, like increasing convection areas, adding forced air through the circuit, or using heat pipes with coolant. These approaches may add exterior components, which translates into more volume and/or more power needed for the conversion. Alternative methods are based on packaging techniques. These techniques are applied at both the semiconductor level and in the arrangement of physical components [3], [4], i.e. embedded passive integrated circuits, power module integrated lead frame, planar integrated LCT (same package for several reactive components i.e. inductors, capacitors), etc. Device temperature is a critical parameter in determining the electrical behavior of power devices because these devices dissipate a considerable amount of power (heat) and are often operated in high temperature environments. Many parameters of these devices are temperature dependent and are responsible for the overall performance of the system. Traditional microelectronic semiconductor models (SPICE, Saber) have the ability to include temperature dependence in their models, but the temperature used by the semiconductor must be chosen prior to the simulation. Thus, temperature remains constant at the predetermined value during the entire simulation. Using this approach will result in an inaccurate model, since these type of devices heat up significantly due to the power dissipated within the device (self-heating) and the power dissipated by adjacent devices (thermal coupling). Therefore, while the circuit's initial behavior temperature dependence may be accounted for, after operating it over a sufficiently long period of time, i.e. enough to stabilize temperature conditions, the initial model is not necessarily valid anymore.

There has been significant effort to model thermal effects on the electronic behavior of components (electro-thermal behavior) [5], [6], etc. The main goals for these models are to achieve high precision, accurate temperature characterization, and simulation efficiency. Most of the methods used to model the electro-thermal behavior of a component feature a thermal representation of the device in the electrical model [6]. This representation consists of multiple RC (resistance-capacitance) thermal networks, where power losses in the components are represented as sources of heat, and ambient temperature is set as the "zero reference." The RC network represents each volume of the device and therefore acts as a physical representation only. Thus, the $\mathrm{RC}$ values must be calculated based on each volume geometry and material property. As a result, the electrical model is represented as a temperature function.

Thermal nodes in the network can feed back the resulting temperature to the electrical model. Most of these approaches

\footnotetext{
${ }^{1}$ This work is supported by U.S. Army Research Laboratory under Cooperative Agreement W911NF-04-2-0033
} 
target the component level, since the geometry as well as the environment specifications are well defined; i.e. single TO247 IGBT with heatsink TTC1406 [8], a TO220AB power MOSFET [5], etc. The dynamic behavior of power electronic components can also be simulated using the same RC thermal networks as part of the simulation profile, provided that all thermal networks are well known [9].

\section{RELEVANCE}

Switch mode power supplies (SMPS) that convert variable DC voltages to controlled DC voltage levels are widely used due to their high efficiency and versatility for power conversion. Although modeling these types of circuits is not an easy task, due to their non-linearity, accurate models have been developed over the years [10], [11], [12] etc. The main objective of these models is to characterize the dynamics of the converter. Therefore, the component parameters used for the models are assumed to be constant. Although this assumption is valid for the dynamic response (electrical time response is on the order of microseconds while temperature response is on the order of seconds) the model lacks accuracy for longer run times.

Power semiconductor devices such as MOSFETS, diodes and transistors are not uniquely temperature dependant; in fact all electronic components are affected by large temperature swings. Some materials offer better thermal stability than others, while other materials cannot withstand high temperature stresses at all. Although the methods mentioned above offer a valid approach to simulating electrical behavior under high temperatures, most of them do not deal with system behavior. For the methods that do support system behavior, the system becomes highly complex and assumes a complete "knowledge" of each piece (i.e. materials, interfaces, geometry) of the system, even though these pieces may not actively participate in the electrical response of the system. As a result, to get a so-called "electro-thermal model" of a DC-DC converter, the designer faces the task of modeling all electrical and all mechanical components to a very detailed level.

This paper proposes an electro-thermal modeling process for power electronic systems that works over a wide range of operating temperatures. Our objectives are to facilitate temperature dependant electrical operation and to obtain thermal profiles at various physical locations. These results are of major importance during the design process, at both the component and system level.

At the system level, thermal management decisions can be made based on temperature profile results. By knowing the location and thermal effect of dissipative and large sized components, a more suitable arrangement of components may be suggested, different materials may be used or alternate cooling method can be selected. At the component level, stress levels vary directly or indirectly with temperature. Case temperatures directly affect junction temperatures, which can be calculated knowing the power dissipated in the component and the package thermal resistance. Indirectly temperature also affects maximum stress voltage and current levels, which define the limits of safe operating areas.

Various component parameters are also affected by temperature such as the forward voltage of diodes, the ESR in capacitors, $\mathrm{R}_{\mathrm{dsON}}$ of MOSFETs, etc. Furthermore, at the system level, the performance of the converter i.e. efficiency, settling point, settling time, etc. is affected by the temperature dependence of each individual parameter.

The method proposed in this paper offers a simplified way to model the electro-thermal behavior of power electronics when thermal steady state has been reached. The outcomes are summarized as follows:

- A simplified method for a steady state system electrothermal model of power electronics has been developed. The approach is based on integrating thermal model simulation results with an electrical model. The proposed approach is based on discrete interactions between the thermal and electrical models, making the finalized electro-thermal simulation much simpler than simulating an electro-thermal model.

- Simulation results for a typical application, showing thermal as well as electric performance are presented. The differences between a standard electrical model and electro-thermal modeling are discussed.

- The accuracy and complexity of the newly proposed electro-thermal models are analyzed and compared to previously proposed approaches. The modeling approach is suitable for electro-thermal characterization and provides both electrical and thermal characterization of power electronic systems.

\section{System Electro-Thermal UNIFIED MODEL}

The proposed electro-thermal model can be simplified using the diagram showed in Fig. 1.

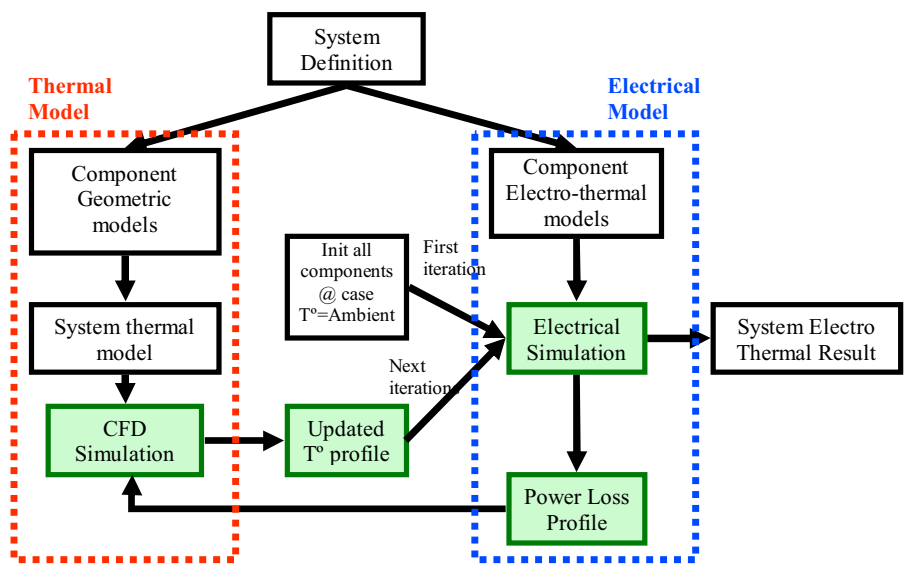

Figure 1. System electro-thermal model 
Step 1: System Definition.

As shown in Fig. 1, the first step in the proposed electrothermal modeling method is to define the system. Specifically for SMPS applications, the size of the board, selection of electrical components, heatsink dimensions and interface materials must be specified.

Step 2: Thermal Model.

Using the geometric description of the largest and most thermally dissipative electrical devices, geometric models are derived. Components such as the board and heat-sinks should also be included. For simplicity, basic three dimensional figures are preferred. Each component model should have a defined thermal conductivity; this property is obtained from datasheets or from the material properties. Subsequently, the components are connected (mechanically) into a complete three dimensional system to create a system thermal model. The universe for the thermal model should be defined as a large box (compared to our system) containing all the components. Finally, all the universe walls are setup at the given ambient temperature.

Using the resulting system thermal model with individual power loss as an input for each modeled device, the thermal model can then be simulated using a computational fluid dynamics (CFD) software. Forced air may also be included in the simulation by adding air flow at certain velocity on one or more walls. The result of the simulation is a thermal profile at every physical location on the system.

Step 3: Electrical Model.

The purpose of these steps is to develop an electrical model for the system that depends on a given temperature profile. In order to do so, a temperature dependant model for the parameters of the electrical components should be developed. Although all parameters are affected by temperature, the intent is to simplify the model. Therefore, the parameters modeled are the ones which are most affected by wide temperature variations. By including these temperature dependant parameter models in the basic electrical model of the component, we obtain the component electro-thermal model, which as a result will have an extra input; case temperature. It is useful to also have as an output of this model the power that is dissipated in the component, since power dissipation determines thermal behavior.

The electrical model is completed by connecting all components for the given circuit. Using a setup that includes input voltages, output power, etc, a first electrical simulation can be achieved using all components with their respective case temperatures at ambient temperature. As a result, a power loss profile for the main components affecting the thermal behavior of the system can be obtained. The power loss for the main components is feed back to the system thermal model, and after running a new CFD thermal simulation, an updated case temperature profile can be obtained, which is used as the temperature input for the next electrical simulation. The result yields a better approximation of the system electro-thermal behavior. These steps should be repeated until the power loss and thermal profiles show low levels of variations.

\section{A. Thermal Model}

Based on the system definition (component selection), simple geometric models of components, specifically those affecting the thermal behavior of the system, are built. Geometric models should contain the physical description of each component: package, material and size. It is important to take into account the components that do not have power losses such as; the printed circuit board, heat-sinks, and large components with almost no power dissipation, should also be included in this model. Although they do not dissipate power, they affect the thermal behavior of the system.

All these components are then placed together consistent with an "appropriate structure". Finally, all the mechanical/electrical components that may interfere or affect the heat flow of the system are added to the resultant model, which we call "System Thermal Model".

\section{B. Electrical Model}

This subsection contains the description of the electrical modeling process as a temperature dependant system. The objective of these steps is to model the electrical components and parameters affected by large temperature swings.

Every electrical component (passive, discrete and magnetic) exhibits thermally dependant electrical behavior, where some are affected more than others. Thermal stress affects not only the electrical behavior of components, but also their reliability (life time). Reliability is a figure of merit that is hard to determine precisely, due to the many factors and levels of dependency. Furthermore, this characteristic is valuable if it is defined for every component in the system, since the reliability number of the system is determined by the weakest component.

Electrical models for individual components can be obtained directly from manufacturer's datasheets and/or from experimental testing. The idea is not to have the most accurate mathematical model of temperature dependence, but to obtain an approximate "lookup table" for the most important parameters of the modeled components that are thermally dependant over a well defined operating temperature range.

\section{System Electro-Thermal Simulation}

In general for SMPS, we can assume that thermal response is much slower than electrical response. In other words, if there is a change in the electrical system, i.e. line or load variations, the electrical model starts responding at the same time. While the electrical system changes, which induces changes in the power loss profile, the thermal state also changes but at a much slower rate. In general, the thermal system reaches 
steady state long after electrical steady state is reached. Based on this assumption, we can visualize two systems interacting in a discrete way. Electrical variations cause changes in power loss, which, at the same, time cause thermal system change. Since the electrical system is affected by temperature variations, it should be adjusted accordingly with the modified thermal profile.

Furthermore, thermal simulation based on initial power loss values, usually gives a good first step temperature profile (approximation) for the thermal behavior of the system. But in order to obtain more accurate system characteristics like output voltage, efficiency, etc., the electrical response should be adjusted accordingly. If the power loss profile noticeably changes due to the thermal variation, then the first approximation is not accurate and another adjustment (iteration) is required.

\section{APPLICATION EXAMPLE}

A typical power supply for an automotive application is taken as a case of study for this paper. The specifications for the power converter are derived from a hybrid electric vehicle requirement, and are as follows:

- Input voltage $600 \mathrm{Vdc}(500 \mathrm{~V}-680 \mathrm{~V})$

- Output voltage $28 \mathrm{Vdc}$

- Output power $100 \mathrm{~W}$

- Operating temperature $85^{\circ} \mathrm{C}$

- Maximum board area of about $60 \mathrm{~mm}$ x $60 \mathrm{~mm}$

- Isolation Input/Output 3000V

- Isolation input-output/baseplate $1500 \mathrm{~V}$

Due to the isolation requirement, a transformer is required to deliver power to the load. It is well known that most commercially available surface mount switches (MOSFET) are rated at a voltage below $1000 \mathrm{~V}$ with current values measuring up to 200A. For the required levels of DC input voltage, the selection of the primary switches will depend mostly on the off-voltage stress experienced by these transistors. For this reason, the half bridge topology and its derivatives offer the best option for this specific application.

\section{Simulation RESUlts}

\section{A. Electrical model}

Assuming that all components in the system start at the nominal ambient temperature, and that thermal response time is much larger than electrical response time, it is reasonable to initialize all temperature dependant electrical models at the nominal ambient temperature. For the given application, the steady state power loss for each main power loss component is calculated at specific line and load conditions. The result is the first power loss profile (i.e. first iteration) based on the nominal ambient temperature.

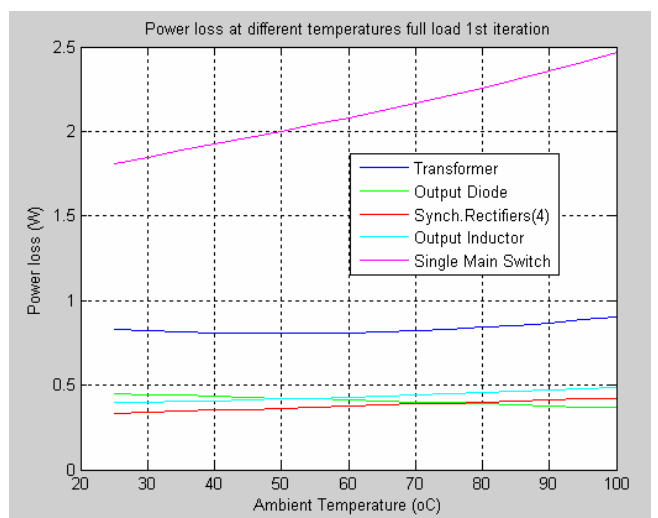

Figure 2. First iteration electrical model-Power loss profile at different ambient Temperatures and full load

Figure 2 and Figure 3 show the dependence of power loss, per component, at different ambient temperature and load conditions.

The results obtained from the electrical model at the given conditions can then be feed back to the thermal model.

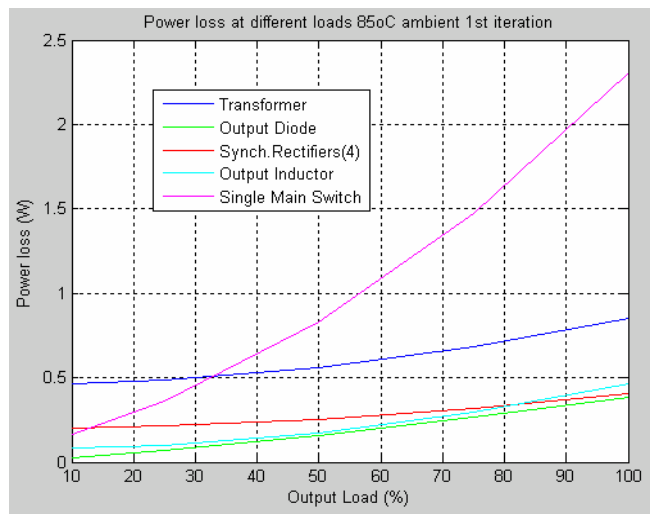

Figure 3. First iteration electrical model-Power loss profile at different loads $85^{\circ} \mathrm{C}$ ambient temperatures

\section{B. Thermal model:}

Given the temperature and size requirements for this application, it is necessary to separate the high power components (requiring high power dissipation) from the control circuitry (requires low power dissipation). Since the high power components will need to dissipate more heat, it is important to use special material and cooling mechanisms (i.e. heatsink) to maintain the temperatures within tolerance levels.

Figure 4 shows the thermal model for the half-bridge converter using Coolit, a thermal simulation software package. Notice from the figure that each component has been drawn in its simplest form and as previously mentioned, the control circuitry (control board) is separated from the power circuitry (power board).

Figure 5 shows the main components on the power board, each one with its respective geometric and material specification. 


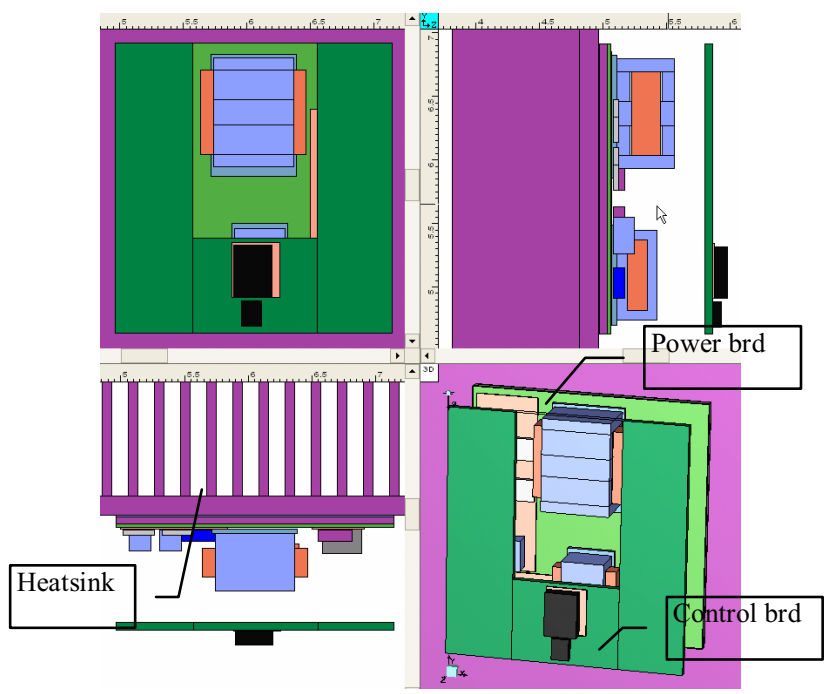

Figure 4. Thermal model, power and control brd plus heatsink

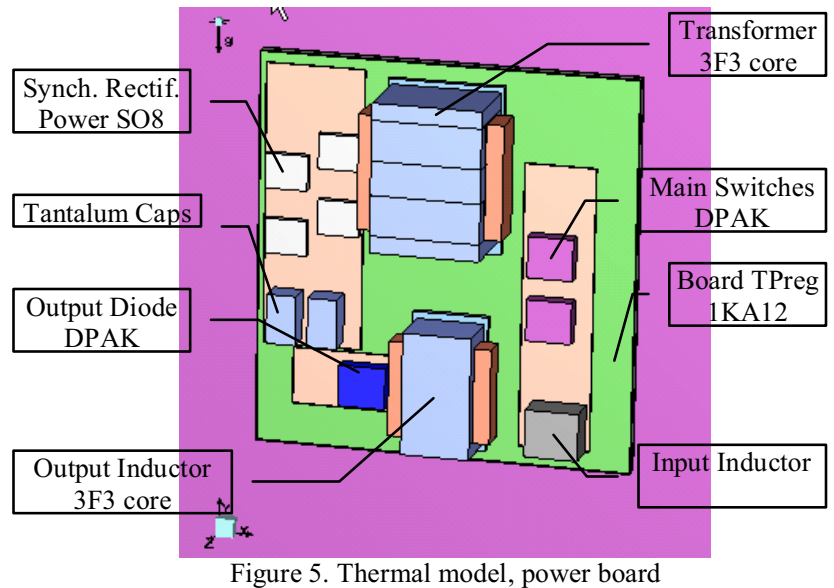

As an example, Figure 6 shows the thermal model for the main transformer core. Notice that the material specifications such as thermal conductivity, density, etc. are also considered.

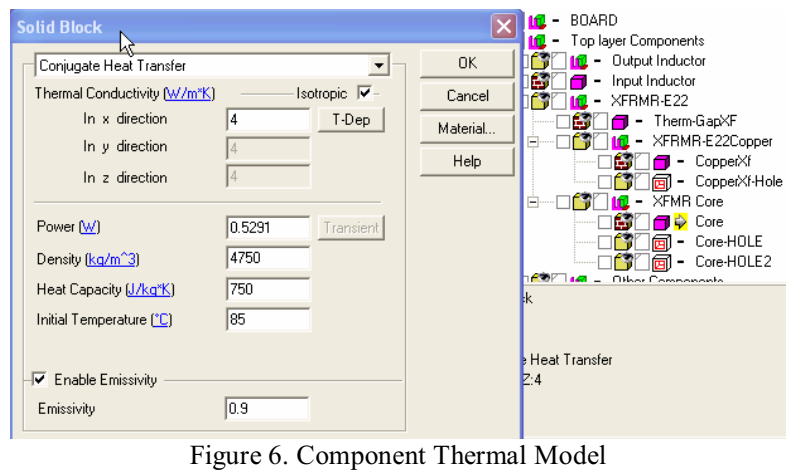

\section{Thermal simulation:}

This step is performed using Coolit, a CFD software simulation tool, used to predict the temperature profile of the converter. Figure 7 and Figure 8 show the thermal simulation of the model drawn in Figure 4 (first iteration). This simulation was used with the power loss profile obtained from MATLAB, a computing software tool, at full load and $85^{\circ} \mathrm{C}$ ambient temperature.

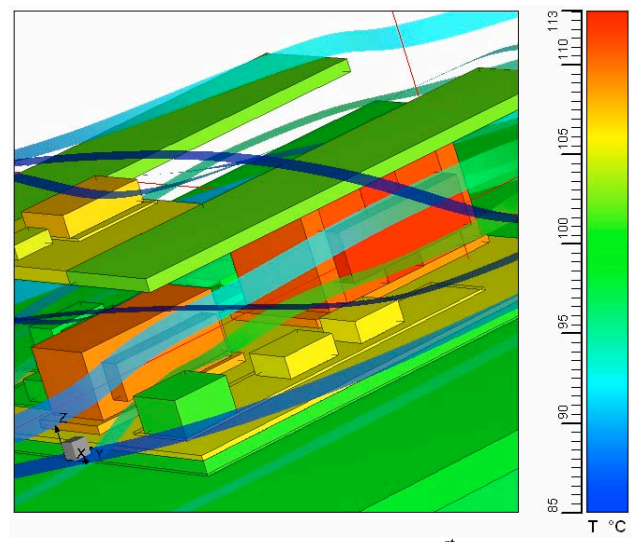

Figure 7. Thermal simulation, $1^{\text {st }}$ iteration

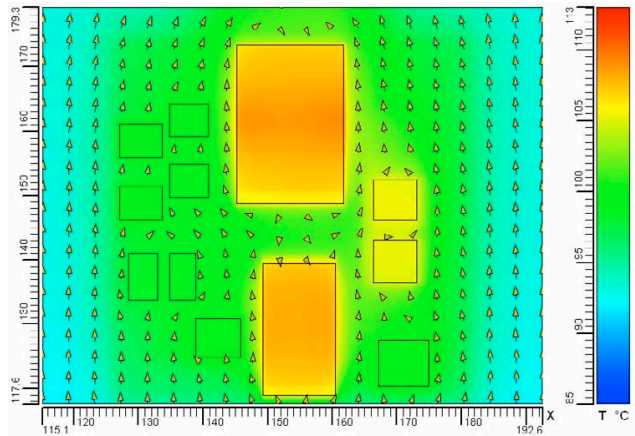

Figure 8. Thermal simulation. Cross sectional area on Power board

After exporting the result files from Coolit to MATLAB, a new temperature profile was obtained and a second power calculation was performed using the MATLAB model "second iteration". This power profile is feed back to the thermal model to obtain second iteration thermal results. For this particular application example three iterations were required to obtain less than $1 \%$ variation on the thermal profiles.

It can be observed from the simulation results that the transformer is experiencing the highest temperature rise. This is because all the power loss on the transformer is dissipated using just its convective area. Since the transformer is not placed directly on the power board, the power board-heatsink arrangement cannot "help" with its heat dissipation. At the same time the control board does not have any components in close vicinity of the transformer, which keeps them relatively thermally isolated. As a result, the control board does not undergo a significant temperature change, which makes the controller circuitry more thermally uniform

Note the following observation; although there is no forced air in the system, air is moving within the "universe" due to the temperature difference between the components. Figure 8 shows this movement, where even the direction of the fins in the heatsink affects the thermal response of the system. 
Figure 9 and Figure 10 show the thermal simulation results for a power loss profile at full load and $85^{\circ} \mathrm{C}$ ambient temperature after three iterations.

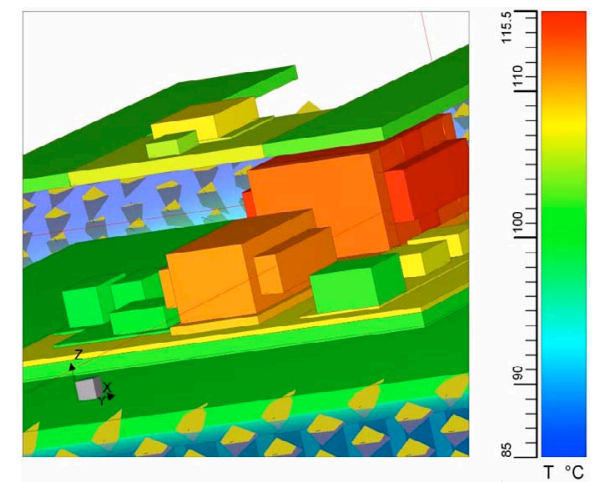

Figure 9. Thermal simulation results, after $3^{\text {rd }}$ iteration

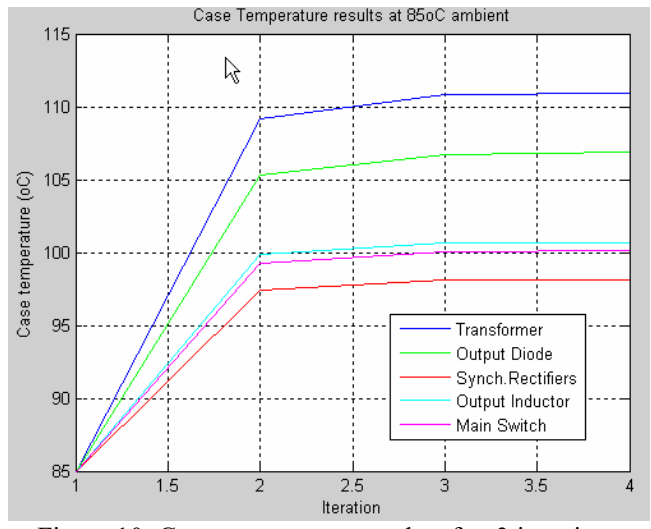

Figure 10. Case temperature results after 3 iterations

Table 1 presents both the experimental results and simulation predictions after three iterations.

TABLE 1

COMPARISON BETWEen TEMPERATURES ObTaINED By SimUlation Results (Matlab-CoOlit) And Experimental Testing

\begin{tabular}{|c|c|c|}
\hline Component & Simulation Results $\left({ }^{\circ} \mathrm{C}\right)$ & Experimental $\left({ }^{\circ} \mathrm{C}\right)$ \\
\hline Output Inductor & 110.9 & 103.51 \\
\hline Transformer & 111.6 & 123.58 \\
\hline Switches & 106.8 & 111.85 \\
\hline Out Capacitors & 98.1 & 100.15 \\
\hline Diode rectifier & 100.7 & 100.95 \\
\hline Synch rectifier & 100.1 & 106.92 \\
\hline PWM IC & 106.7 (without heatsink) & 101.85 (heatsink) \\
\hline
\end{tabular}

\section{CONCLUSIONS}

- Using a simple thermal model in conjunction with a temperature dependant electrical model, an electro-thermal characteristic for a DC-DC converter system is achieved.

- Using the proposed approach, the thermal profile is simultaneously an output of the electro-thermal model. This data is important when installing the system in the final location, since it can affect and be affected by diverse factors external to the DC-DC converter. For example if there are thermally sensitive components close by, they could be affected by the load placed in the DC-DC converter, or if there is another subsystem forcing air into the DC-DC converter, the system will be thermally coupled.

- Using the thermal results, a reliability analysis could be developed based on the thermal stress of each component.

- Thermal management of the DC-DC converter is more effectively accomplished with the thermal profile and heat dissipation outputs of the CFD simulation.

\section{REFERENCES}

[1] Ohadi, M.; Jianwei Qi; "Thermal management of harshenvironment electronics". IEEE Semiconductor Thermal Measurement and Management Symposium, pp. 231 - 240 March 2004

[2] De Jong, E.C.W.; Ferreira, J.A.; Bauer, R.; "Evaluating thermal management efficiency in converters". IEEE Power Electronics Specialists Conf., vol. 6, pp. 4881 - 4887, June 2004.

[3] Popovic, J.; Ferreira, J.A.; Van Horck, F.B.M.; "Evaluating packaging effectiveness in power electronics". IEEE Power Electronics Specialists Conf., vol. 2, pp. 881 - 886. June 2003

[4] Hopkins, D.C.; Mathuna, S.C.O.; Alderman, A.N.; Flannery, J.; "A framework for developing power electronics packaging". Applied Power Electronics Conference and Exposition. vol. 1, pp. 9-15. 15-19 Feb. 1998.

[5] Jakopovic, Z.; Sunde, V.; Bencic, Z., "Electrothermal modeling and simulation with SIMPLORER", IEEE International Conf. on Industrial Technology, vol. 2, pp. 1141-1145, Dec. 2003.

[6] Azar, R.; Udrea, F.; Ng, W.T.; Dawson, F.; Findlay, W.; Waind, P.; Amaratunga, G., "Advanced electrothermal Spice modelling of large power IGBTs", IEEE Proceedings Circuits, Devices and Systems, vol. 151, Issue: 3, pp. 249-253. June 2004.

[7] Schepp, O.; Lenz, M.; "A versatile electrothermal model of an integrated full bridge device taking into account various boundary conditions". Applied Power Electronics Conference and Exposition, vol. 1, pp. 185-190. Feb. 1997.

[8] Hefner, A.R.; Blackburn, D.L.; "Simulating the dynamic electrothermal behavior of power electronics circuits and systems". IEEE Transactions on Power Electronics, vol. 8, Issue: 4, pp. 376-385. Oct. 1993.

[9] Schroder, S.; De Doncker, R.W.; "Physically based models of high power semiconductors including transient thermal behavior". IEEE Transactions on Power Electronics, vol. 18, Issue 1, pp. 231-235, Jan. 2003.

[10] R. D. Middlebrook and S. Cuk; "A general unified approach to modeling switching converter power stages", IEEE Power Electronics Specialist Conf., 1976 Proceedings pp.18 - 34.

[11] Lehman, B.; Bass, R.M.; "Switching frequency dependent averaged models for PWM DC-DC converters", IEEE Power Electronics Specialists Conf., vol. 11, pp. 89-98. January 1996.

[12] Jian Sun; Rajasekaran, V.; "Switching DCM analysis and modeling of half-bridge converters with current-doubler rectifier", IEEE Power Electronics Specialists Conf. vol. 1, pp. 384-389. June 2001. 